\title{
Caractérisation Physico-Chimique Des Eaux De Surface Dans Un Environnement Minier Du Centre-Ouest De La Côte d'Ivoire : Cas Du Département De Divo
}

\author{
Yao Kouassi Serge Aristide,
}

Doctorant, Laboratoire des Sciences du Sol, de l'Eau et des Géomateriaux Ahoussi Kouassi. Ernest, Maître de Conférences

Laboratoire des Sciences du Sol, de l'Eau et des Géomateriaux (LSSEG)/ Unité de Formation et de Recherche (UFR) des Sciences de la Terre et des Ressources Minières (STRM)/ Université Felix Houphouët Boigny, Abidjan

Doi:10.19044/esj.2020.v16n12p293 URL:http://dx.doi.org/10.19044/esj.2020.v16n12p293

\section{Résumé}

Les ressources en eaux de surface du département de Divo sont soumises à de fortes pressions anthropiques suscitées par le développement. Pour ce faire, cette étude a été initiée pour déterminer les caractéristiques physico-chimiques des eaux du département et celles situées à proximité des sites miniers. A cet effet, treize paramètres physicochimiques ont été étudiés. Ce sont les ions majeurs $\left(\mathrm{Ca}^{2+}, \mathrm{Mg}^{2+}, \mathrm{Na}^{+}, \mathrm{K}^{+}, \mathrm{Cl}^{-}, \mathrm{SO}_{4}{ }^{2-}, \mathrm{HCO}_{3}^{-}\right), \mathrm{pH}, \mathrm{Eh}$, température, turbidité, conductivité électrique (EC) et $\mathrm{NO}_{3}^{-}$. Les ions analysés et les paramètres de terrain ont été comparés aux normes des eaux brutes. Les résultats ont été traités d'une part à l'aide de la méthode hydrochimique (diagramme de piper) pour déterminer la typologie des eaux et d'autre part à l'aide de l'analyse statistique multivariée (l'Analyses en Composantes Principales Normée (ACPN)), pour mettre en évidence l'origine de la minéralisation des eaux. Les résultats montrent que la plupart des eaux sont voisines de la neutralité. Les concentrations en nitrates sont parfois élevées, elles ont pour valeur maximale $164,8 \mathrm{mg} / \mathrm{l}$. Les eaux sont moyennement minéralisées avec une conductivité moyenne de $608,4 \mu \mathrm{S} / \mathrm{cm}$. Les eaux à proximité des sites d'exploitation industriels sont les plus minéralisées. Ces eaux sont fortement caractérisées par un hydrofaciès de type chloruré calcique et magnésien. Les phénomènes à l'origine de la minéralisation des eaux de Divo sont essentiellement liés à l'agriculture et aux rejets urbains. Cette étude nous a permis de caractériser les paramètres physico-chimiques des eaux de surface du département. Une étude plus détaillée doit être réalisée afin de caractériser avec précision l'origine de la minéralisation des eaux. 
Mots clés : Activité minière, Eaux de surface, Qualité, Physico-chimique, Minéralisation

\title{
Physico-Chemical Characteristic of Surface Waters in Mining Environment of the Central West of Ivory Coast: Case of the Department of Divo
}

\author{
Yao Kouassi Serge Aristide,
}

Doctorant, Laboratoire des Sciences du Sol, de l'Eau et des Géomateriaux

Ahoussi Kouassi. Ernest, Maître de Conférences

Laboratoire des Sciences du Sol, de l'Eau et des Géomateriaux (LSSEG)/

Unité de Formation et de Recherche (UFR) des Sciences de la Terre et des Ressources Minières (STRM)/ Université Felix Houphouët Boigny, Abidjan

\section{Abstract}

The surface water resources of Divo's department are subjected to strong anthropic pressures caused by the development. With this intention, this study was initiated to determine the physicochemical characteristics of water of the department and those located near the sites mines. To this end, thirteen physicochemical parameters were studied. These are the major ions $\mathrm{CCa}^{2+}$, $\left.\mathrm{Mg}^{2+}, \mathrm{Na}^{+}, \mathrm{K}^{+}, \mathrm{Cl}^{-}, \mathrm{SO}_{4}{ }^{2-}, \mathrm{HCO}_{3}{ }^{-}\right), \mathrm{pH}$, Eh, temperature, turbidity, electrical conductivity (EC) and $\mathrm{NO}_{3}{ }^{-}$. The analyzed ions and the field parameters were compared with the standards raw water. The results were treated on the one hand using the hydrochemic method (diagram of piper) to determine the typology of water and on the other hand using the multivariate statistical analysis (Normed Principal component Analysis (NPCA) to underline the origin of the mineralization of water. The results show that most of the waters are close to neutrality. The nitrate concentrations are sometimes high, they have as a maximum value $164.8 \mathrm{mg} / \mathrm{l}$. water is moderately mineralized with an average conductivity of $608.4 \mu \mathrm{S} / \mathrm{cm}$. water near the mining sites is the most mineralized. This water is strongly characterized by a hydrofacies of the calcic and magnesian chlorinated type. The phenomena behind the mineralization of water of Divo are mainly linked to agriculture and urban rejections. This study enabled us to characterize the physicochemical parameters of surface water of the department. A more detailed study must be carried out in order to characterize precisely the origin of the mineralization of water. 
Keywords: Mining activity, Surface water, Quality, Physico-chemical, Mineralization

\section{Introduction}

L'industrie minière constitue un secteur essentiel pour le développement économique de nos pays. Elle joue un rôle important dans le développement de nombreux pays partout dans le monde (États-Unis, Canada, Australie, Russie, Brésil, Afrique du Sud, Chine, UE, etc.) et continue d'être un contributeur important aux économies nationales et régionales (MINEO, 2000). Cependant cette activité, comme la plupart des activités humaines (l'agriculture, la production de déchets résidentiels et industriels), déverse des métaux dissous et particules dans les sols environnants, des rivières et des sédiments qui peuvent avoir un impact significatif sur non seulement la qualité mais aussi la durabilité des ressources en eau (Othmani et al., 2015). On considère souvent les activités minières comme une source majeure de dégradation de l'environnement (Omanović et al., 2015 ; Othmani et al., 2015). Aujourd'hui, la connaissance du secteur minier est fondée sur des exemples passés de dégradation de l'environnement et d'impact sur la santé humaine (Durucan et al., 2006). En conséquence, les activités minières sont de plus en plus contestées par les populations locales et les organisations de protection de l'environnement (Yao, 2018). L'industrie minière crée aussi de l'emploi et de la richesse. Pour ces raisons, de nombreux projets miniers ont été réalisés au cours des dernières décennies, principalement dans les pays africains.

Le rejet d'effluents d'extractions minières se fait dans les cours d'eau environnant des sites miniers, sans aucune mesure adéquate pouvant entrainer une modification de la chimie de ces eaux et donc une possible pollution, contraignant les populations à se passer de cette eau mal saine. Malheureusement, ces ressources ont été soumises à plusieurs problèmes géo environnementaux, principalement la pollution des eaux de surface. Les travaux de Akatumbila et al. (2016) ; Lakhili F et al. (2016) et Ngouala et al. (2016) ont tous montré que la qualité des eaux de surface est toujours influencée par la présence des activités anthropiques.

L'exploitation minière est depuis un certain temps très développés en Côte d'Ivoire. Le département de Divo, situé au Centre-Ouest de la Côte d'Ivoire est l'une des principales provinces métallogéniques du pays. On y compte plusieurs sites miniers industriels, en cours d'exploitation, plus précisément à Hiré et Agbaou. Dans le département plusieurs lentilles aurifères ont été identifiées (Yapi., 2014), dont certaines sont en exploitation par les sociétés minières telles que : Andeavour Mining et Agbaou Gold mine. Les effluents issus de l'extraction du minerai sont déversés dans l'environnement, principalement dans les eaux à proximité des sites miniers sans aucune mesure 
de traitement. Cette situation entraine un risque de contamination et/ou de pollution des eaux situées dans le voisinage des sites d'exploitation. Cette étude, réalisée dans le cadre du réseau international Amedée a pour objectif d'évaluer ou déterminer la qualité des eaux de surface du département à travers la caractérisation des paramètres physico-chimiques.

\section{Présentation de la zone d'étude}

\subsection{Situation géographique}

Situé au Centre-Ouest de la Côte d'Ivoire, le département de Divo est à environ $187 \mathrm{~km}$ d'Abidjan, la capitale économique. Il couvre une superficie d'environ $3577 \mathrm{~km}^{2}$ et est situé entre la latitude $05^{\circ} 40^{\prime}$ et $06^{\circ} 10^{\prime}$ 'Nord et la longitude $05^{\circ} 30^{\prime}$ et $04^{\circ} 40^{\prime}$ Ouest (Figure 1). Le département compte (07) sous-préfectures dont : la sous-préfecture de Chiepo, d'Hiré, de Zégo, de Didoko, de Nébo, d'Ogoudou et de Divo. Il est limité par le département d'Oumé au Nord, le département de Lakota à l'Ouest, le département de Grand Lahou au Sud-Est, le département de Guitry au Sud-Ouest et le département de Tiassalé à l'Est. Il est constitué de trois sous bassins versants : le bassin versant de Bandama, du Gô et le bassin versant de Boubo, avec la présence de deux grandes zones minières à Hiré et à Agbaou. Le climat de la zone de Divo est celui de l'attiéen au régime équatorial qui est un climat équatorial de transition. Ce climat est caractérisé par quatre saisons : une grande saison sèche entre décembre et avril, une grande saison de pluies de mai à juillet, une petite saison sèche d'août à septembre et une petite saison des pluies d'octobre à novembre. Mais ces dernières années, cette répartition saisonnière est totalement perturbée ; On observe ainsi qu'une seule saison de pluie d'avril à octobre, suivie d'une saison sèche de novembre à mars. Ce changement climatique perturbe sérieusement le calendrier agricole et aura un impact négatif sur les productions agricoles, particulièrement sur les cultures vivrières. La pluviométrie moyenne mensuelle du département varie entre 232,59 mm en juin (grande saison des pluies) à 20,39 mm en janvier (grande saison sèche). 


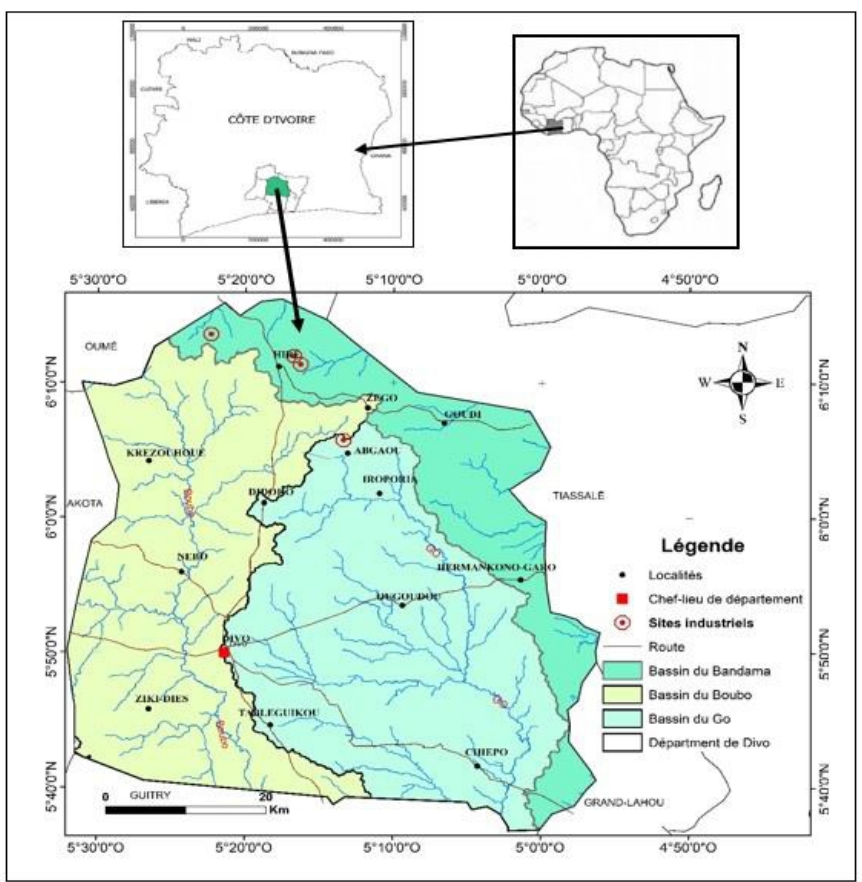

Figure 1. Localisation de la zone d'étude

\subsection{Les activités minières dans le département de Divo}

Le département regorge d'énorme ressource minière surtout dans la commune de Hiré et dans la localité d'Agbaou. Ces ressources sont exploitées industriellement par les entreprises étrangères telles que : NEWCREST, ANDEAVOUR et aussi de manière clandestine appelée orpaillage (Figure 2). L'exploitation industrielle de l'or en Côte d'Ivoire se fait à travers une exploitation à ciel ouvert. Ce mode d'exploitation génère de gros problèmes environnementaux notamment la destruction du sol, la pollution des cours d'eau, l'assèchement des nappes souterraines, la destruction des végétaux...etc.
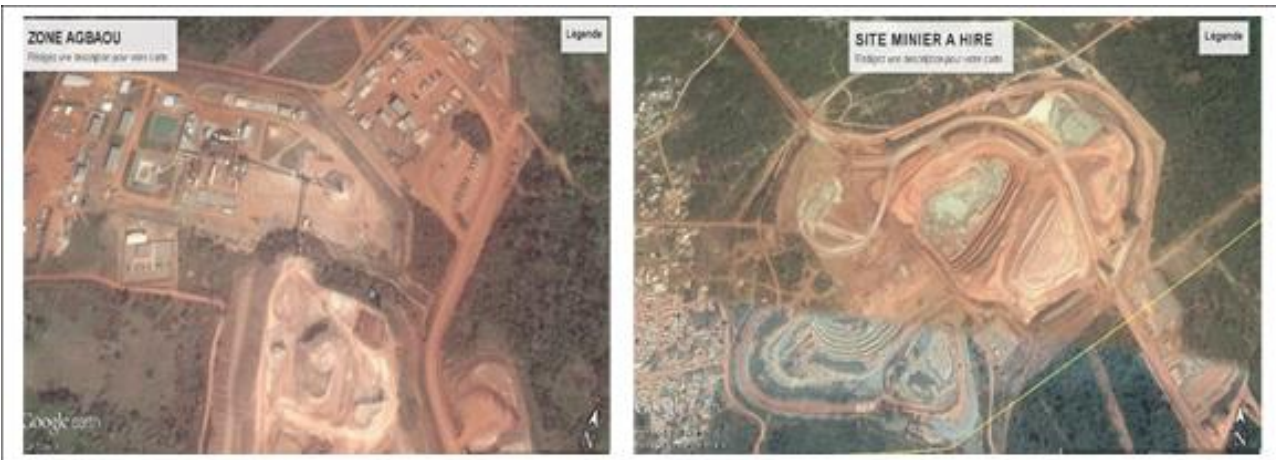

Figure 2 : Mine à ciel ouvert à AGBAOU et HIRE (Source : Newrest Mining, Avril 2012 ; Google map, 2016 


\subsection{Contexte géologique et hydrogéologique}

\subsubsection{Contexte géologique}

Le département est constitué essentiellement par un socle ancien qui regroupe trois types de formations : les formations archéennes (migmatites), les formations libériennes (granitoïdes) et les formations birimiennes (conglomérats, grès et schistes) (PRICI, 2016).

\section{Formations libériennes}

Les formations libériennes sont constituées essentiellement de granitoïdes. Ce sont les métagranites à biotite, les métagranodiorites à biotite ou hornblende, les métadiorites et métatonalites ;

\section{Formations birimiennes}

Dans le nord du département on distingue des métabasaltes, des amphiboles, des métadolérites, micaschistes et des métagabbros (autour de la ville d'Hiré), des métarhyolites, des métadacites, des tufs acides, des brèches, des schistes chloriteux et des sédiments associés (Figure 3).

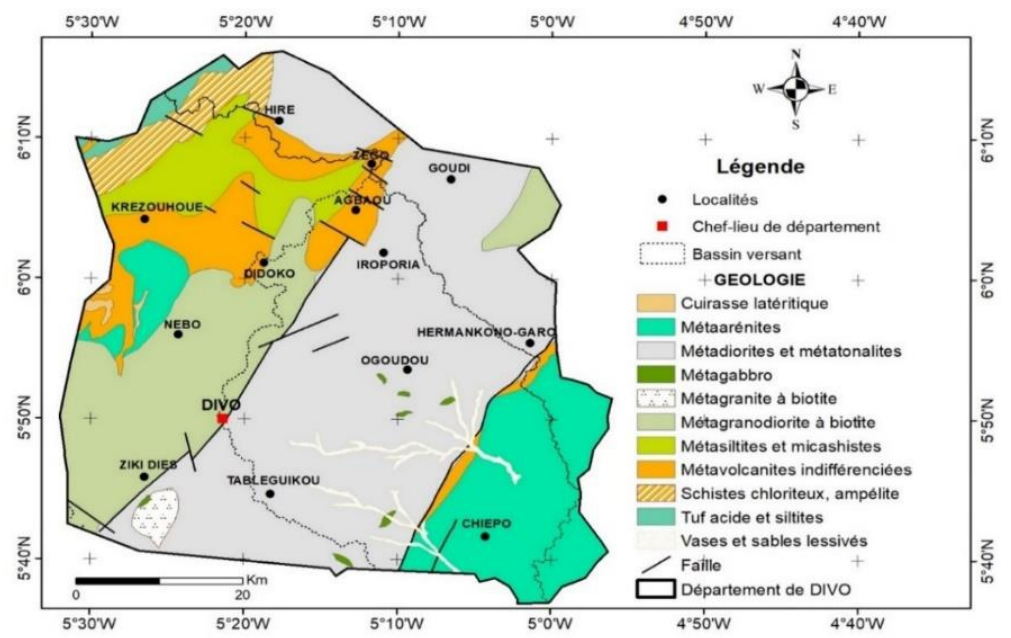

Figure 3 : Carte géologique de la zone d'étude

\subsubsection{Contexte hydrogéologique}

On distingue deux types d'aquifères profonds : les aquifères continus du bassin sédimentaire côtier et les aquifères discontinus du socle.

\section{Aquifères continus}

Les aquifères continus sont localisés dans les formations du bassin sédimentaire côtier et comprennent deux nappes superposées ; la nappe du continental terminal et la nappe des sables quaternaires (Adiaffi,2009). 


\section{Aquifères discontinus}

Les aquifères discontinus couvrent la plus grande partie du département, constitués d'aquifères d'altérites et d'aquifères de substratum fissurés.

\section{Matériel et méthodes}

\subsection{Méthodes d'échantillonnage et d'analyse}

L'échantillonnage des eaux du département a été effectué en septembre 2018, ce qui correspond à la saison pluvieuse. L'échantillonnage a été effectué sur les cours d'eau du département et à proximité des sites miniers. 27 échantillons d'eau ont été prélevés dont 8 sur le bassin de boubo, 10 sur le bassin de Gô et 9 sur le bassin de Bandama. En somme 19 échantillons d'eau ont été prélevés sur les deux bassins qui abritent les sites miniers en exploitation. Ces échantillons ont été prélevés sur les deux cours d'eau permanent du département (le Boubo et le Gô) et leurs différents affluents. Les échantillons d'eau prélevés ont été mis dans des bouteilles en polyéthylène de capacité $500 \mathrm{ml}$, préalablement lavées à l'acide nitrique puis à l'eau distillée. Sur le terrain, avant le remplissage des bouteilles, celles-ci ont été lavées trois fois avec l'eau à prélever. Le remplissage des bouteilles a été fait à ras bord, puis le bouchon vissé afin d'éviter tout échange gazeux avec l'atmosphère. Les échantillons d'eau ont été conservés à l'abri de la lumière et de la poussière dans une glacière à $4^{\circ} \mathrm{C}$, puis transportés au laboratoire pour analyse. Les paramètres tels que : la température, le $\mathrm{pH}$, la conductivité électrique $(\mathrm{CE})$ et le potentiel redox (Eh) ont été mesurés in situ à l'aide d'un multi-paramètre de type WTW 3110. Les analyses chimiques réalisées au laboratoire de l'IRD de l'Université Nangui Abrogoua ont concerné $\left(\mathrm{Ca}^{2+}\right),\left(\mathrm{Mg}^{2+}\right),\left(\mathrm{Na}^{+}\right),\left(\mathrm{K}^{+}\right)$, $\left(\mathrm{Cl}^{-}\right),\left(\mathrm{HCO}_{3}{ }^{-}\right)\left(\mathrm{SO}_{4}{ }^{2-}\right)$ et l'ion $\left(\mathrm{NO}_{3}^{-}\right)$. Ces éléments ont été analysés à l'aide d'équipements adéquats constitué d'un spectrophotomètre de laboratoire de type HACH/DR 6000 et des méthodes d'analyses préconisées par Rodier et al. (2009). Les résultats d'analyse ont ensuite été comparés aux valeurs des eaux brutes (Arrêté du 11/01/07 relatif aux limites et référence de qualité des eaux brutes). Les points d'échantillonnage sont présentés par la figure 4. 


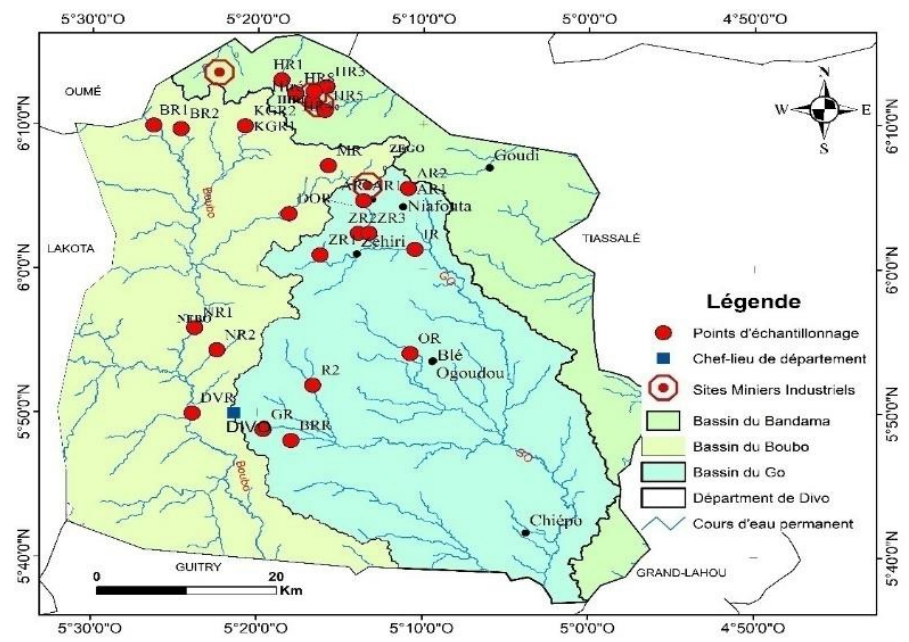

Figure 4 : Carte de la répartition des points d'échantillonnage des eaux de surface

\subsection{Traitement des données}

\subsubsection{Balance ionique}

La balance ionique est une méthode de validation, elle est utilisée pour vérifier la cohérence des résultats obtenus au laboratoire.

$B I=\frac{\sum\left(\text { Cations }-\sum(\text { Anions })\right)}{\sum\left(\text { Cations }+\sum(\text { Anions })\right)} \times 100$

$\mathrm{BI}=$ Balance Ionique en $\%$;

[ ] = Concentration exprimée en meq/l.

En règle générale, les résultats des analyses sont considérés de la manière suivante :

- excellent lorsque $\mathrm{BI}<5 \%$;

- acceptable lorsque $5 \%<\mathrm{BI}<10 \%$;

- douteuse lorsque $\mathrm{BI} \geq 10 \%$.

Une analyse chimique des eaux n'est considérée comme représentative et acceptable que lorsque la balance ionique est inférieure ou égale à $10 \%$ (Kouassi et al., 2013).

\subsubsection{Caractérisation hydrochimique des eaux}

Les faciès chimiques sont couramment utilisés en hydrogéologie pour la description de la composition des eaux naturelles. La nécessité d'une comparaison aisée, voire une classification hydrochimique des eaux naturelles nécessite l'utilisation des représentations graphiques. Dans notre étude, La démarche graphique qui a été retenue et utilisée est le diagramme de piper. Ce diagramme est souvent utilisé dans les études hydrochimiques par plusieurs auteurs dont Bashir et al. (2017) et Salifu et al. (2017). En Côte d'ivoire, cette méthode est largement utilisée par Biemi (1992) et Ahoussi (2008). 


\subsubsection{Traitement statistique des données}

L'approche statistique qui a été utilisée pour étudier les phénomènes à l'origine de la minéralisation des eaux est basée sur l'Analyse en Composantes Principales Normées (ACPN); C'est une méthode statistique multidimensionnelle permettant de synthétiser les informations dans le but de comparer les systèmes entre eux. Son application dans l'étude hydrochimique a été réalisée par plusieurs auteurs (Akatumbila et al, 2016) ; Ahoussi et al, 2018), pour expliquer d'une part les ressemblances chimiques entre les différentes eaux et/ou les différents pôles d'acquisition de la minéralisation et d'autre part les variables qui gouvernent ces mécanismes. L'ACPN a été réalisée sur vingt-sept (27) échantillons d'eau de surface. Cette analyse porte sur 13 paramètres : la température $(\mathrm{T})$, la turbidité (Turb), $\mathrm{pH}$, Conductivité Electrique (CE), le potentiel redox (Eh), Potassium $\left(\mathrm{K}^{+}\right)$, Calcium $\left(\mathrm{Ca}^{2+}\right)$, Sodium $\left(\mathrm{Na}^{+}\right)$, Magnésium $\left(\mathrm{Mg}^{2+}\right)$, Chlorures $\left(\mathrm{Cl}^{-}\right)$, Sulfates $\left(\mathrm{SO}_{4}{ }^{2-}\right)$, Bicarbonates $\left(\mathrm{HCO}_{3}{ }^{-}\right)$, Nitrates $\left(\mathrm{NO}_{3}{ }^{-}\right)$. L'analyse statistique a été réalisée à l'aide du logiciel STATISTICA 7.1.

\section{Résultats}

\subsection{Balance ionique}

La balance ionique établit pour confirmer la justesse des analyses, est dite satisfaisante car $71 \%$ des eaux de surface ont une balance ionique satisfaisante. Elle est acceptable car 9\% des eaux de surface sont dites acceptables.

\subsection{Caractéristiques physico-chimiques des eaux}

Les résultats des analyses statistiques des données physico-chimiques des eaux de surface du département sont présentés dans le tableau I.

Tableau I : Analyse statistique des paramètres physico-chimiques des eaux du département

\begin{tabular}{|c|c|c|c|c|c|c|c|}
\hline $\begin{array}{l}\text { Paramètres } \\
\text { physico- } \\
\text { chimiques }\end{array}$ & Unités & $\begin{array}{l}\text { Minimum } \\
\text { (M) }\end{array}$ & $\begin{array}{l}\text { Maximum } \\
(\mathrm{m})\end{array}$ & $\begin{array}{l}\text { Moyenne } \\
(\mu)\end{array}$ & $\begin{array}{l}\text { Ecart- } \\
\text { type }(\alpha)\end{array}$ & $\begin{array}{l}\mathrm{Cv}= \\
(\alpha / \mu)\end{array}$ & $\begin{array}{l}\text { Norme Eaux } \\
\text { brutes }\end{array}$ \\
\hline $\mathrm{T}$ & ${ }^{\circ} \mathrm{C}$ & 24,1 & 30,3 & 26,7 & 1,21 & 0,04 & $<25$ \\
\hline $\mathrm{pH}$ & & 6,53 & 7,9 & 7,26 & 0,35 & 0,05 & $6,5<\mathrm{pH}<8,5$ \\
\hline $\mathrm{CE}$ & $\mu \mathrm{S} / \mathrm{Cm}$ & 183,2 & 1909 & 608,74 & 480,64 & 0,79 & $<250$ \\
\hline Eh & $\mathrm{mg} / \mathrm{l}$ & $-43,2$ & 28,3 & $-10,66$ & 18,45 & $-1,7$ & \\
\hline Turb & $\mathrm{mg} / \mathrm{l}$ & 5,36 & 111 & 27,17 & 24,22 & 0,89 & 0,5 \\
\hline $\mathrm{Ca}^{2+}$ & $\mathrm{mg} / \mathrm{l}$ & 18 & 172 & 53,55 & 42,56 & 0,79 & 100 \\
\hline $\mathrm{Na}^{+}$ & $\mathrm{mg} / \mathrm{l}$ & 2,49 & 31,49 & 8,29 & 6,44 & 0,77 & $<200$ \\
\hline $\mathrm{Mg}^{2+}$ & $\mathrm{mg} / \mathrm{l}$ & 4,37 & 98,41 & 27,84 & 21,97 & 0,78 & $<50$ \\
\hline $\mathrm{K}^{+}$ & $\mathrm{mg} / \mathrm{l}$ & 0,7 & 37,4 & 8,57 & 9,67 & 1,12 & $<10$ \\
\hline $\mathrm{Cl}^{-}$ & $\mathrm{mg} / \mathrm{l}$ & 0,4 & 14,8 & 4,9 & 3,4 & 0,69 & $<200$ \\
\hline $\mathrm{SO}_{4}{ }^{2-}$ & $\mathrm{mg} / \mathrm{l}$ & 0 & 53 & 8,62 & 14,4 & 1,62 & $<250$ \\
\hline $\mathrm{HCO}_{3}^{-}$ & $\mathrm{mg} / \mathrm{l}$ & 2,4 & 21,6 & 8,13 & 4,83 & 0,59 & \\
\hline $\mathrm{NO}_{3}^{-}$ & $\mathrm{mg} / \mathrm{l}$ & 1,2 & 164,8 & 15,07 & 40,58 & 2,69 & $>50$ \\
\hline
\end{tabular}




\subsection{Caractéristiques physiques des eaux}

\subsubsection{Le pH}

Les résultats montrent que la plupart des eaux sont relativement neutres avec des valeurs de $\mathrm{pH}$ qui varient entre 6,53 à la station DVR et 7,93 à la station AR1(tous deux situés sur le sous bassin versant de Gô) avec une moyenne de 7,26. Les stations à $\mathrm{pH}$ situé entre 7,1 et 7,9-représentent plus de $74 \%$, ainsi les valeurs du $\mathrm{pH}$ des eaux sont assez homogènes ( $\mathrm{Cv}$ est très faible 0,05). La répartition spatiale du $\mathrm{pH}$ des eaux est illustrée par la figure 5 .

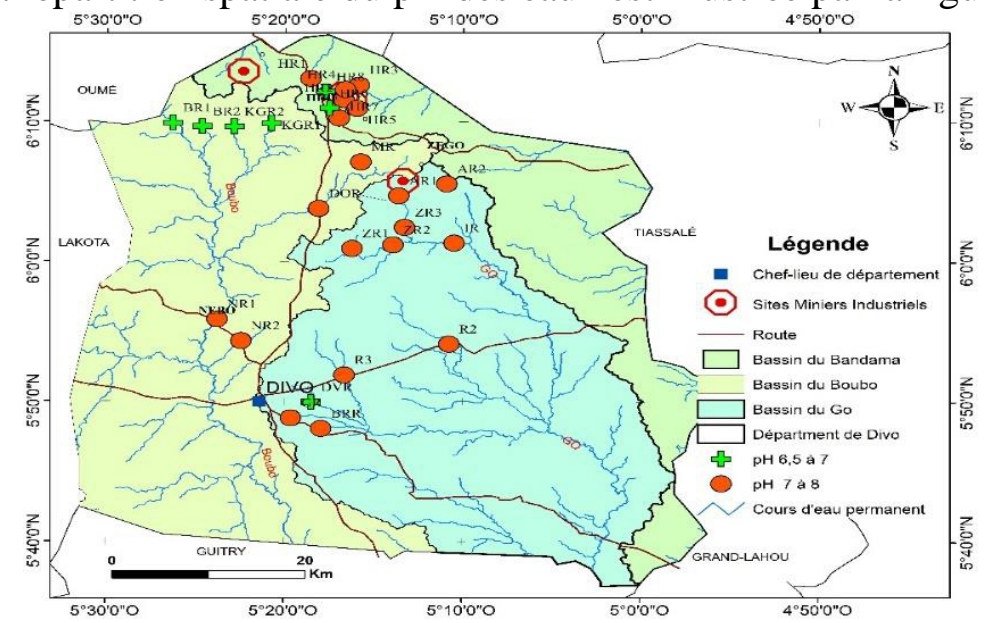

Figure 5 : Carte de répartition spatiale du $\mathrm{pH}$ des eaux du département de Divo

\subsubsection{Température des eaux}

La température enregistrée varie entre $24,1^{\circ} \mathrm{C}$ (station $\mathrm{MR}$, rivière située sur la route de la mine d'Agbaou à $2 \mathrm{~km}$ de la mine sur le bassin de boubo) et $30,3{ }^{\circ} \mathrm{C}$ (station HR7, eau de surface utilisée pour les activités d'orpaillage sur le bassin de bandama), avec un écart type de $1,21^{\circ} \mathrm{C}$ et une moyenne de $26,7^{\circ} \mathrm{C}$. Les valeurs les plus fréquentes sont situées entre 25 et 27 ${ }^{\circ} \mathrm{C}$. Cet intervalle représente plus de $81 \%$.

\subsubsection{Conductivité électrique (CE)}

Les valeurs de la conductivité électrique varient de $183,2 \mu \mathrm{S} . \mathrm{cm}^{-1}$ (station DVR qui est le site de prélèvement de la SODECI de Divo sur le bassin de boubo) à $1909 \mu \mathrm{S} . \mathrm{cm}^{-1}$ (station HR5, rivière située à $70 \mathrm{~m}$ d'un site minier à Hiré sur le bassin de Bandama), avec une moyenne de $608,74 \mu{\mathrm{S} . \mathrm{cm}^{-1}}^{-1}$ (Figure 6). Les valeurs de la conductivité électrique des eaux sont dispersées, comme l'indique le $\mathrm{Cv}$ (environ 0,79). Cependant, cette gamme peut se découper en 3 classes : $48 \%$ des échantillons ont une conductivité électrique inférieure à $400 \mu \mathrm{S} / \mathrm{cm}$, donc elles sont faiblement minéralisées ; celles ayant une CE comprise entre 400 et $1000 \mu \mathrm{S} / \mathrm{cm}$ représentent $37 \%$ des échantillons (moyennement minéralisée) et seulement 15\% des eaux ont une CE supérieure 
à $1000 \mu{\mathrm{S} . \mathrm{cm}^{-1}}^{-1}$ (fortement minéralisées). Les points d'eau HR1, HR2, HR3, HR4 et HR5 qui sont tous situés à proximité des sites miniers du bassin versant de Bandama enregistrent des valeurs de conductivité très élevées.

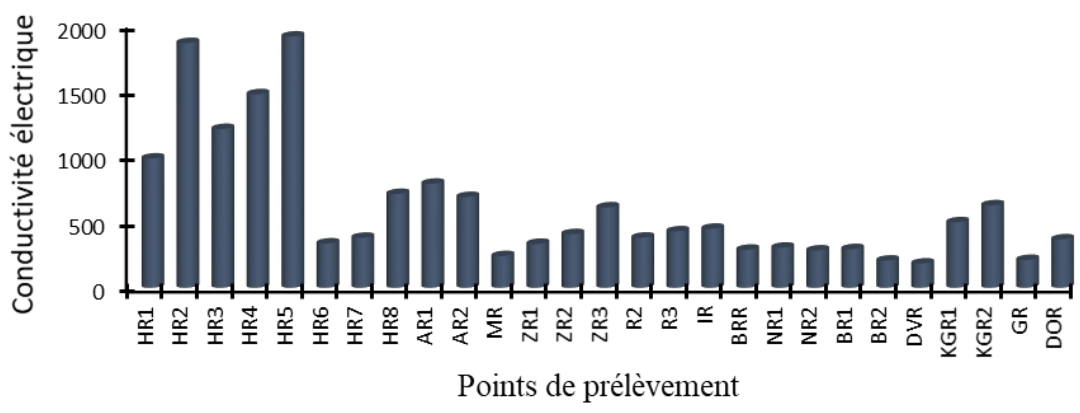

Figure 6 : Evolution spatiale de la conductivité électrique des eaux du Département de Divo

La carte de répartition spatiale des conductivités des eaux montre que les eaux des stations (HR1, HR2, HR3, HR4, HR5 du bassin versant de bandama) et (AR1 du bassin de Gô), situées à proximité des zones minières sont fortement minéralisées (figure7).

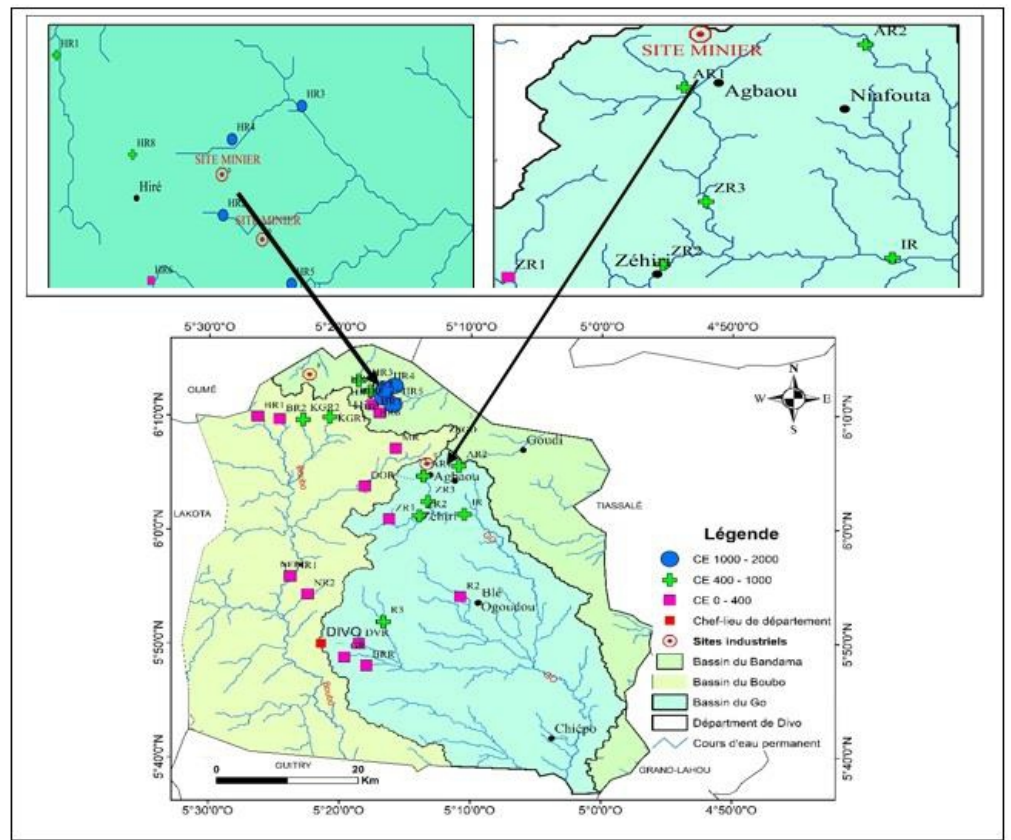

Figure 7 : Carte de répartition spatiale de la conductivité des eaux du département de Divo

\subsubsection{Concentration en anions $\left(\mathrm{HCO}_{3}{ }^{-} \mathrm{Cl}^{-}\right.$et $\left.\mathrm{SO}_{4}{ }^{2-}\right)$}

La concentration en bicarbonate dans les eaux de surface du département varie entre un minimum de $2,4 \mathrm{mg} / \mathrm{l}$ à la station BRR enregistré 
au niveau du bassin du Gô, cette eau est située près du village de Brabodoukou et un maximum de $21,6 \mathrm{mg} / \mathrm{l}$ au point d'eau HR4 enregistré au niveau du bassin du Bandama, avec une teneur moyenne de $8,13 \mathrm{mg} / \mathrm{l}$.

Les concentrations en sulfates enregistrées dans les eaux sont très faibles. Elles oscillent entre 0 et $53 \mathrm{mg} / \mathrm{l}$. Les valeurs élevées ont été mesurées au niveau des rivières (HR3 et HR4) sur le bassin versant du Bandama, rivière sortant de la mine située à $100 \mathrm{~m}$ du pit et (AR1), eau en provenance de la mine d'Agbaou, mais prélevée dans une plantation cacaoyère au niveau du bassin du Gô (30 mg/l).

Les chlorures varient de $3,4 \mathrm{mg} / \mathrm{l}$ à $14,8 \mathrm{mg} / \mathrm{l}$, avec une moyenne de $4,93 \mathrm{mg} / \mathrm{l}$. Les teneurs comprises entre 2 et $7 \mathrm{mg} / \mathrm{l}$ représentent $82 \%$ des échantillons. La concentration maximale $(14,8 \mathrm{mg} / \mathrm{l})$ a été enregistrée au point d'eau (HR5) sur le bassin du Bandama. Les différents histogrammes de ces anions sont représentés sur la figure 8. L'ion sulfate est l'anion le plus abondant, avec une moyenne de 8,62 mg/l. Les anions majeurs se présentent selon l'ordre d'abondance suivant : $\mathrm{SO}_{4}{ }^{2-}>\mathrm{HCO}_{3}>\mathrm{Cl}^{-}$.

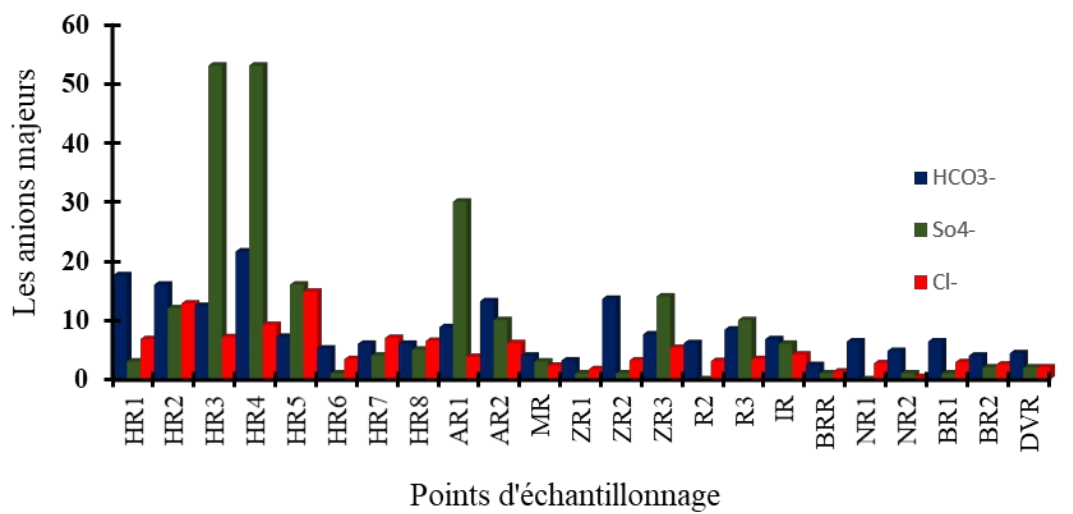

Figure 8 : Concentration des anions $\mathrm{HCO}_{3}{ }^{-}, \mathrm{Cl}^{-}$et $\mathrm{SO}_{4}{ }^{2}$ dans les eaux du département de Divo

\subsubsection{Concentration en cations $\left(\mathrm{Ca}^{2+}, \mathrm{Na}^{+}, \mathrm{Mg}^{2+}\right.$ et $\left.\mathrm{K}^{+}\right)$}

Le calcium est le cation le plus abondant. La concentration du calcium dans les eaux de surface oscille entre 18 et $172 \mathrm{mg} / \mathrm{l}$, avec une moyenne de $53,55 \mathrm{mg} / \mathrm{l}$. Les teneurs en magnésium varient de 4,37 à 35,73 mg/l, avec une moyenne de 27,84 mg/l. Quant au sodium les teneurs varient de 2,49 à 31,49 $\mathrm{mg} / \mathrm{l}$. Les concentrations en potassium sont les plus faibles. Elles varient de 0,7 à $37,4 \mathrm{mg} / \mathrm{l}$, avec une moyenne de $8,57 \mathrm{mg} / \mathrm{l}$ (Figure 9 et 10 ). 


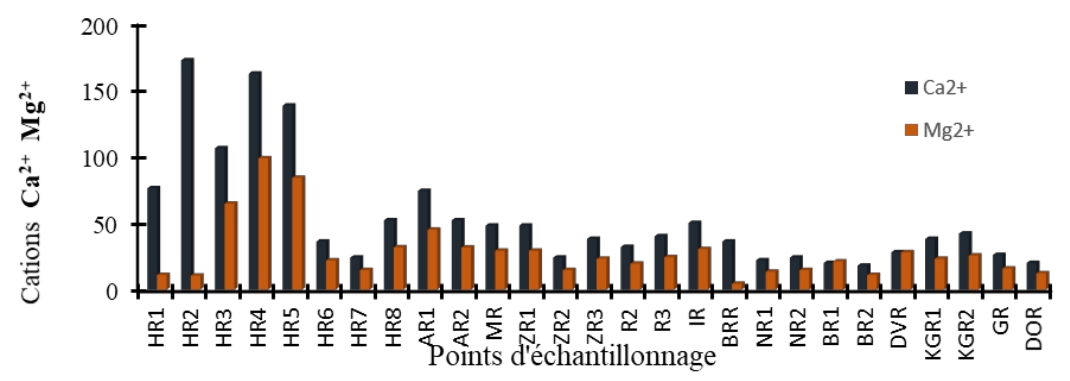

Figure 9 : Concentration des cations $\mathrm{Ca} 2+$ et $\mathrm{Mg} 2+$ dans les eaux du département de Divo

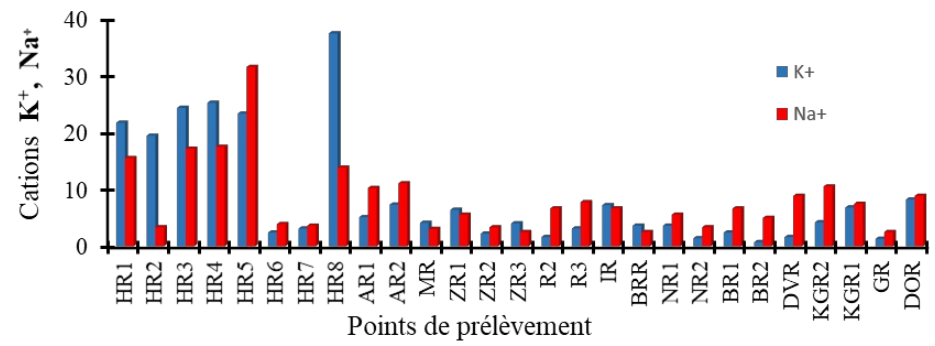

Figure 10 : Concentration des cations $\mathrm{K}^{+}$et $\mathrm{Na}^{+}$dans les eaux du département de Divo

\subsubsection{Concentration en nitrate $\left(\mathrm{NO}_{3}{ }^{-}\right)$}

L'azote est présent dans les eaux sous sa forme nitrate $\left(\mathrm{NO}_{3}{ }^{-}\right)$. Les valeurs de ce paramètre dans les eaux échantillonnées sont très variables et oscillent entre $1,2 \mathrm{mg} / \mathrm{l}$ (station ZR1, rivière traversant des champs de riz et de cacaoyer) et $164,8 \mathrm{mg} / 1$ (station HR5, rivière située à $70 \mathrm{~m}$ d'un site minier sur le bassin de bandama, cette rivière est utilisée pour les bovins) (Figure 11) avec une moyenne de $15,07 \mathrm{mg} / \mathrm{l}$. Les valeurs les plus fréquentes sont comprises entre 2 et $8 \mathrm{mg} / \mathrm{l}$. Les points d'eau HR5 et HR2 situés à proximité des sites miniers présentent respectivement les concentrations les plus élevées $164,8 \mathrm{mg} / \mathrm{l}$ et $144,6 \mathrm{mg} / \mathrm{l}$.

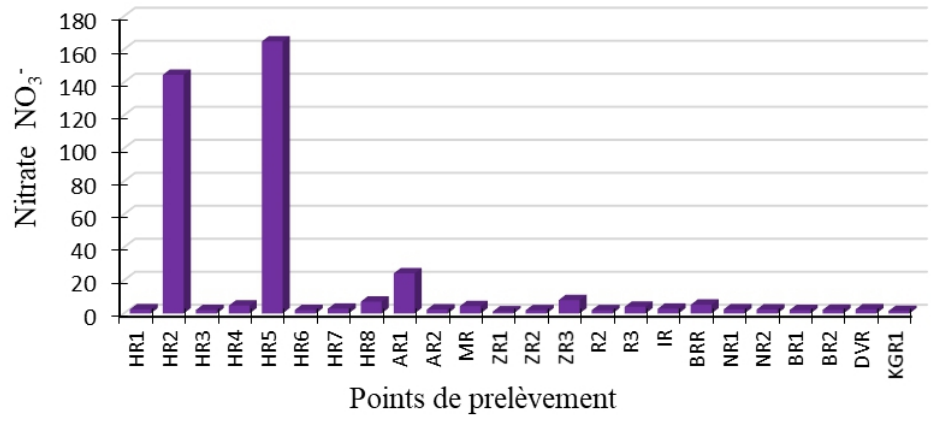

Figure 11 : Concentration du nitrate dans les eaux du département de Divo 


\subsection{Faciès chimique des eaux}

$\mathrm{La}$ représentation des résultats des analyses chimiques sur le diagramme de Piper (Figure 12) montre que le facies chimique dominant est le chloruré calcique et magnésien $(85 \%)$ des échantillons d'eau. Il est caractérisé par une prédominance des chlorures sur les bicarbonates pour les anions et des alcalinoterreux sur les alcalins pour les cations.

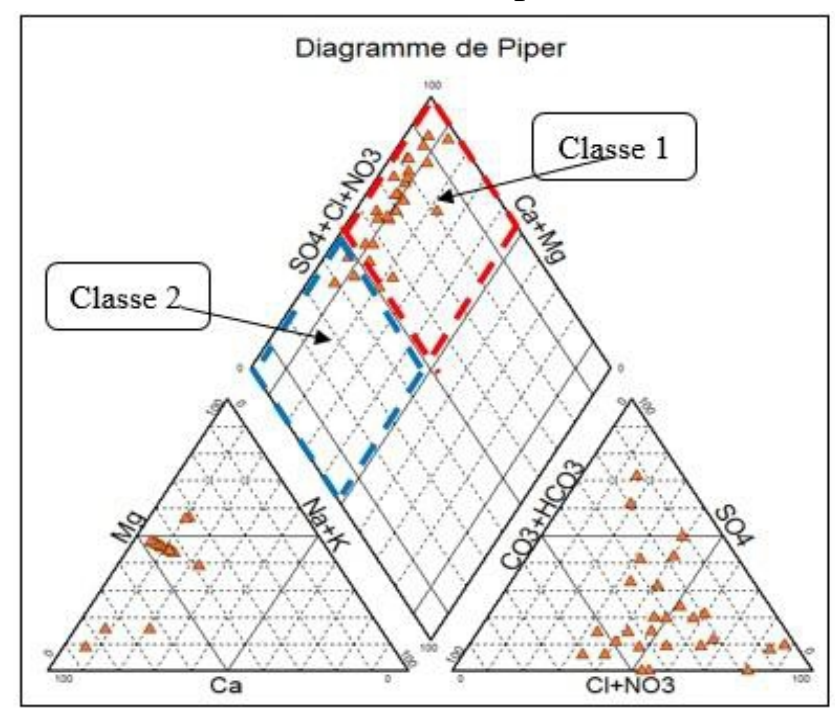

Figure 12 : Classe hydrochimique des eaux de surface dans le diagramme de Piper

\subsection{Résultats de l'étude statistique multivariée Analyse en Composantes Principales (ACP)}

Les résultats de l'ACP sont résumés ci-dessous. Le tableau des valeurs propres (Tableau II) montre que les trois premiers facteurs représentent 70,53\% de la variance exprimée. Ces facteurs regroupent le maximum de la variance exprimée et sont suffisants pour traduire exactement l'information recherchée.

Tableau II : Valeurs propres et pourcentages exprimés par les axes principaux

\begin{tabular}{ccccc}
\hline Valeur numéro & Val Propre & $\begin{array}{c}\% \text { Total } \\
\text { variance }\end{array}$ & $\begin{array}{c}\text { Cumul Val } \\
\text { Propre }\end{array}$ & Cumul \% \\
\hline 1 & 5,75 & 41,13 & 5,75 & 41,13 \\
2 & 2,60 & 18,57 & 8,35 & 59,71 \\
3 & 1,51 & 10,82 & 9,87 & 70,53 \\
\hline
\end{tabular}

La matrice de corrélation qui présente les différentes corrélations entre les variables nécessaires pour la compréhension des phénomènes étudiés est présentée par le tableau III. 
Tableau III : Matrice de corrélation entre les variables

\begin{tabular}{|c|c|c|c|c|c|c|c|c|c|c|c|c|c|}
\hline Variables & $\mathrm{T}$ & $\mathrm{CE}$ & Turb & $\mathrm{pH}$ & Eh & $\mathrm{Ca}^{2+}$ & $\mathrm{K}^{+}$ & $\mathrm{Mg}^{2+}$ & $\mathrm{Na}^{+}$ & $\mathrm{SO}_{4}^{2-}$ & $\mathrm{Cl}^{-}$ & $\mathrm{HCO}_{3}^{-}$ & $\mathrm{NO}_{3}^{-}$ \\
\hline $\mathrm{T}$ & 1,00 & & & & & & & & & & & & \\
\hline CE & 0,17 & 1,00 & & & & & & & & & & & \\
\hline turb & 0,08 & $-0,26$ & 1,00 & & & & & & & & & & \\
\hline $\mathrm{pH}$ & 0,14 & 0,42 & $-0,06$ & 1,00 & & & & & & & & & \\
\hline Eh & $\overline{0,21}$ & $-0,42$ & 0,01 & $-0,89$ & 1,00 & & & & & & & & \\
\hline $\mathrm{Ca}^{2+}$ & $\begin{array}{c}- \\
0,16\end{array}$ & 0,74 & 0,16 & 0,22 & $\overline{-}, 20$ & 1,00 & & & & & & & \\
\hline $\mathrm{K}^{+}$ & 0,08 & $\mathbf{0 , 7 8}$ & $-0,02$ & 0,13 & $\begin{array}{c}- \\
0,18\end{array}$ & $\mathbf{0 , 7 0}$ & 1,00 & & & & & & \\
\hline $\mathrm{Mg}^{2+}$ & $\overline{-}$ & 0,31 & 0,30 & 0,07 & 0,00 & 0,67 & 0,55 & 1,00 & & & & & \\
\hline $\mathrm{Na}^{+}$ & $0, \overline{26}$ & 0,49 & $-0,08$ & 0,16 & $0, \overline{15}$ & 0,59 & 0,69 & $\mathbf{0 , 7 8}$ & 1,00 & & & & \\
\hline $\mathrm{SO}_{4}^{2-}$ & $\begin{array}{c}- \\
0,10\end{array}$ & $\mathbf{0 , 5 3}$ & 0,31 & 0,24 & $\begin{array}{c}- \\
0,11\end{array}$ & 0,69 & 0,53 & $\mathbf{0 , 8 0}$ & $\mathbf{0 , 5 1}$ & 1,00 & & & \\
\hline $\mathrm{Cl}^{-}$ & 0,12 & 0,64 & 0,05 & 0,21 & $-\overline{27}$ & 0,77 & 0,62 & 0,50 & $\mathbf{0 , 5 8}$ & 0,41 & 1,00 & & \\
\hline $\mathrm{HCO}_{3}^{-}$ & $0, \overline{18}$ & 0,62 & 0,20 & 0,28 & $0, \overline{28}$ & 0,66 & 0,50 & 0,40 & 0,39 & $\mathbf{0 , 5 8}$ & 0,48 & 1,00 & \\
\hline $\mathrm{NO}_{3}{ }^{-}$ & $\begin{array}{c}- \\
0,04 \\
\end{array}$ & 0,50 & $-0,19$ & 0,19 & $\begin{array}{c}- \\
0,18 \\
\end{array}$ & 0,69 & 0,39 & 0,31 & 0,46 & 0,15 & 0,74 & 0,19 & 1,00 \\
\hline
\end{tabular}

Ce tableau donne les différentes corrélations entre les variables. Il existe une forte corrélation entre $\mathrm{CE}$ et $\mathrm{Ca}^{2+}(0,74) ; \mathrm{HCO}_{3}{ }^{-}$et $\mathrm{K}^{+}(0,78)$ et entre $\mathrm{SO}_{4}{ }^{2-}$ et $\mathrm{Mg}^{2+}(0,80)$. Les coefficients de corrélations négatives expriment une opposition entre les variables. Cela signifie que les variables considérées évoluent en sens inverse par rapport au centre.

- Analyse dans l'espace des variables du plan factoriel F1-F2

L'espace des variables du plan factoriel F1-F2 (Figure 13) met en évidence deux groupements de variables suivant l'axe F1. Le premier groupement est constitué par : la $\mathrm{CE}$, les cations $\left(\mathrm{Na}^{+}, \mathrm{Ca}^{2+}\right.$ et $\left.\mathrm{K}^{+}\right)$, les anions $\left(\mathrm{Cl}^{-}, \mathrm{HCO}_{3}^{-}, \mathrm{SO}_{4}{ }^{2-}\right)$ et $\mathrm{NO}_{3}^{-}$dans sa partie négative. Ces regroupements traduisent une minéralisation influencée par les activités anthropique (activités minières). La présence du nitrate $\left(\mathrm{NO}_{3}{ }^{-}\right)$dans ce groupement a une origine superficielle et témoigne d'une pollution anthropique. Cela explique une minéralisation liée aux activités industrielles du département produit à la surface du sol, puis entraînées avec les eaux de ruissellement. De ce fait, le facteur F1 est considéré comme un axe de minéralisation d'origine superficielle (anthropique). Le facteur F2 représente 18,57\% de la variable exprimée, et regroupe les paramètres $\mathrm{T}$ et $\mathrm{pH}$ dans sa partie negative, ainsi que le Eh dans sa partie superieure, la présence du Eh traduit un phénomène d'oxydo-reduction. 


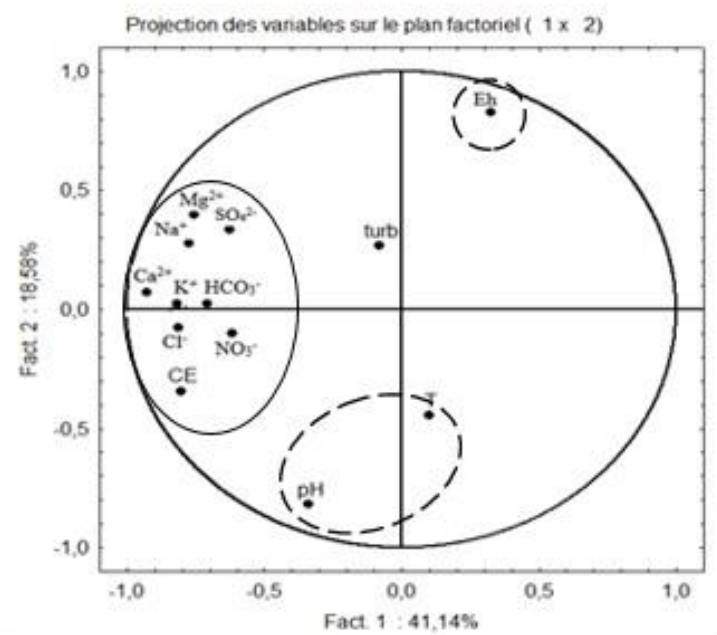

Figure 13 : Analyse dans le plan factoriel F1-F2 des eaux de surface du département

\section{- Analyse dans l'espace des variables du plan factoriel F1-F3}

Le cercle de corrélation entre les composantes F1 et F3 (Figure 14) représente $51,22 \%$ de la variance. Dans le plan factoriel F1-F3, le facteur 1 est toujours déterminé dans sa partie négative par les variables tels que : $\mathrm{Ca}^{2+}, \mathrm{CE}, \mathrm{Na}^{+}$, $\mathrm{K}^{+}, \mathrm{HCO}_{3}^{-}$et $\mathrm{NO}_{3}^{-}$. Il exprime donc le phénomène de la minéralisation anthropique due à la forte corrélation entre $\mathrm{CE}$ et $\mathrm{NO}_{3}{ }^{-}$. En ce qui concerne le facteur 3, il est mis en relief par la Turb qui met en évidence le phénomène des apports superficiels dans les cours d'eaux. Tout comme F1, F3 exprime le phénomène de la minéralisation anthropique lié aux apports superficiels.

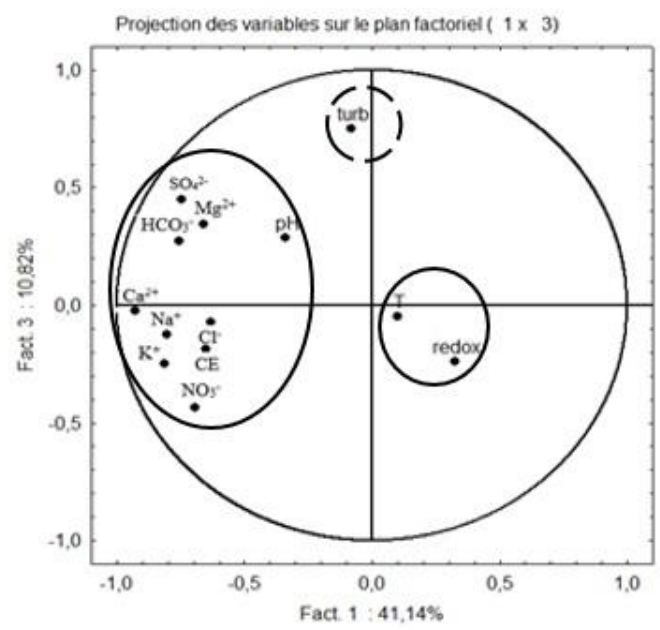

Figure 14 : Analyse dans le plan factoriel F1-F3 des eaux de surface du department 


\section{- Projection des individus dans le plan factoriel F1-F2}

L'étude statistique permet de classer les eaux en trois groupes :

- les eaux fortement minéralisées : Les eaux à proximité des sites miniers et directement influencées par l'exploitation minière. Il s'agit des échantillons des sites HR3, HR2, HR4 et HR5 ;

-les eaux moyennement minéralisées : Ce sont les eaux éloignées des sites d'exploitation minières (HR1, AR2, ZR3) et du site de cyanuration HR8 ;

- les eaux faiblement minéralisées : autres points d'eau qui ne sont pas influencés par les activités minières, elles sont représentées par les échantillons des cours d'eaux HR6, HR7. ZR2, R2, R3, IR, BRR, NR1, NR2, KGR1, DVR, DOR, NR2 et MR (Figure 105).

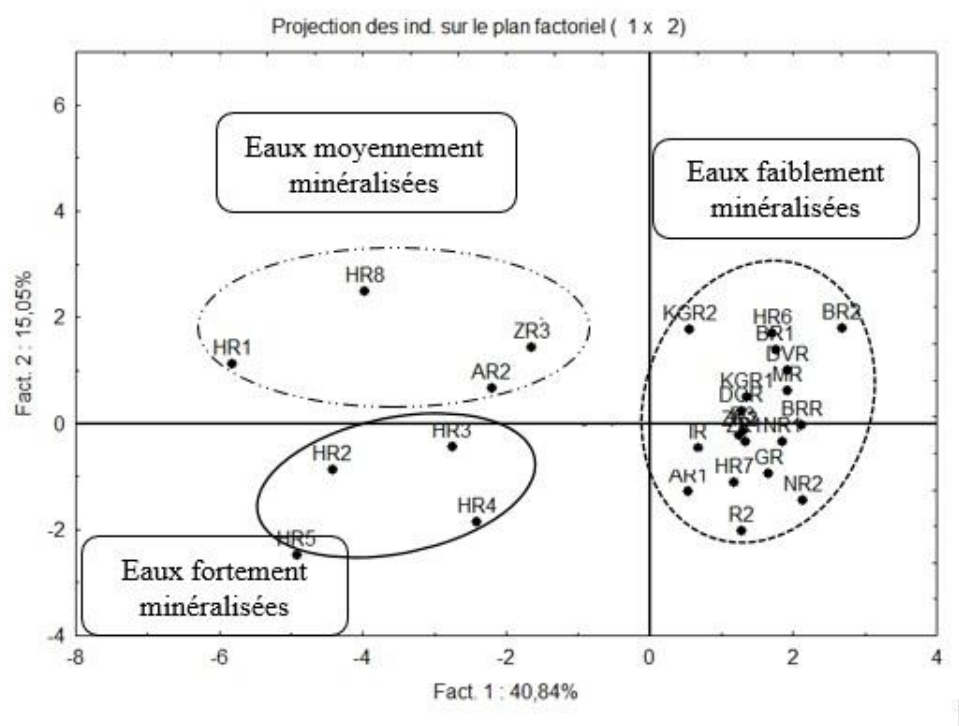

Figure 15 : Espace des unités statistiques du plan factoriel F1×F2 des eaux

\section{Discussion}

\section{Caractéristique physico-chimique}

Les résultats obtenus lors de cette étude ont montré que la température des eaux varie de $24,1^{\circ} \mathrm{C}$ à $30,3^{\circ} \mathrm{C}$ avec une valeur moyenne de $27^{\circ} \mathrm{C}$. Cette gamme de variation est en concordance avec les travaux d'Oga (1998) et Ahoussi et al., (2010) dans les eaux de la région du Grand Abidjan (25,5 à $30,4^{\circ} \mathrm{C}$ ). Ces résultats sont similaires à d'autres travaux sur les eaux de surface tels que Eblin et al., (2014) qui rapportent, qu'en zone tropicale humide, la température moyenne des eaux est environ $30^{\circ} \mathrm{C}$. Selon les travaux de Dudka et al., (1997), lorsque dans un environnement minier la température moyenne des eaux est inférieure à $30^{\circ} \mathrm{C}$, cela pourrait influencer le drainage minier acide (DMA), qui constitue le problème environnemental le plus important de l'industrie extractive. En effet, elle pourrait accélérer les réactions 
d'oxydations des minéraux sulfureux libérant ainsi les métaux présents dans ces minéraux. Ainsi, la réaction d'oxydation de la pyrite contribuant à la formation du DMA et à la libération des métaux s'exprime sous la forme suivante : $2 \mathrm{FeS}_{2}+7 \mathrm{O}_{2}+2 \mathrm{HO}_{2} \quad 2 \mathrm{Fe}^{2+}+4 \mathrm{SO}_{4}{ }^{2-}+4 \mathrm{H}^{+}$. Ces résultats s'accordent avec le travail de Yao., (2018) qui a effectué une étude sur les ressources en eau aux abords du site minier d'Afema $\left(30^{\circ} \mathrm{C}\right)$. Les $\mathrm{pH}$ de ces eaux sont compris entre 6,53 et 7,93 avec une moyenne de 7,26. En effet, cette moyenne du pH montre que les eaux étudiées ne sont pas agressives.

La conductivité électrique varie de 183,2 $\mu \mathrm{S} / \mathrm{cm}$ à $1909 \mu \mathrm{S} / \mathrm{cm}$, avec une moyenne de $608,74 \mu \mathrm{S} / \mathrm{cm}$. Cela montre que les eaux sont en grande partie moyennement minéralisées et donc ne contiennent pas assez de sels minéraux dissous. Ces valeurs de conductivités électriques sont similaires des résultats des études antérieures d'Ahoussi et al., (2010) et Loko et al., (2013). De plus, ces eaux reçoivent les eaux usées domestiques et celles des industries installées en amont, présentant parfois de forte minéralisation, largement supérieur aux valeurs des eaux brutes. C'est le cas des points d'eau HR1 (982 $\mu \mathrm{S} / \mathrm{cm}), \mathrm{HR} 2(1859 \mu \mathrm{S} / \mathrm{cm}), \mathrm{HR} 3(1206 \mu \mathrm{S} / \mathrm{cm}), \mathrm{HR} 4(1470 \mu \mathrm{S} / \mathrm{cm})$ et HR5 (1909 $\mu \mathrm{S} / \mathrm{cm})$. Cette minéralisation élevée dans ces cours d'eau est liée aux activités anthropiques intenses en amont de la zone (activités minières). Eblin et al., (2014) rapportent que l'activité anthropique est un phénomène intervenant dans la minéralisation par les apports superficiels.

\section{Origine des composés azotés (Nitrates)}

Les nitrates constituent l'un des composés azotés dominants dans les eaux du département, en particulier les eaux à proximité des sites miniers. Les teneurs en nitrates observées dans les eaux diffèrent d'un site à l'autre. Cette variabilité spatiale des teneurs en nitrates des eaux traduit également une diversité dans leur origine. Dans une eau naturelle, la minéralisation nitratée peut avoir plusieurs origines.

En effet (Elbaz-Poulichet et al., 2002) stipule que les fortes teneurs en nitrates rencontrées dans les eaux sont dues au lessivage des sols et à la décomposition de la matière organique ou bien liées à la déforestation. Les activités agricoles constituent également une source de production des nitrates. La présence des plantations de bananier, de cacaoyer, de plantation d'hévéa et de palmier à huile dans la zone d'étude et les effets induits (utilisation d'engrais) nous amènent à redouter pour les années avenir des cas de pollution dans cette partie du département.

Les plus fortes teneurs en nitrates dans ces eaux sont beaucoup influencées par les activités anthropiques telles que les activités minières situées parfois à proximité de ces eaux. Les sels nutritifs sont transportés en saison pluvieuse dans les différents cours d'eau par le phénomène de ruissellement. Ces résultats confirment ceux de Tohouri (2012) sur la lagune 
Ono. Les eaux sont sous l'influence d'une part, des pluies qui tombent directement sur les plans d'eau et d'autre part, des eaux de ruissellement. Les pratiques des activités minières et autres activités anthropiques qui se déroulent dans le département participent à la dégradation de la qualité des eaux de surface. En effet, les études de Lakhili et al., (2015) ; Chaouay et al., (2016) et Adjagodo A. et al., (2017) ont montré l'impact des activités anthropiques sur la minéralisation des eaux superficielles. Cette minéralisation est influencée par les sols ferralitiques, qui représentent les caractéristiques du type de sol dans notre zone d'étude. Ils se caractérisent par leur richesse et leur fertilité représentées par les écosystèmes agricoles que sont les bas-fonds marécageux et les plaines alluviales. La zone de Hiré et d'Agbaou appartient au sol ferralitique faiblement desaturé. Le département se caractérise principalement par la présence de trois types de sols : les ferralsols faiblement désaturés sur schistes et micaschistes au Nord, les ferralsols moyennement désaturés au sud de la sous-préfecture de Divo et les ferralsols moyennement désaturés et remanié dans la grande partie du département notament dans les sous-préfectures de Divo, Ougoudou, Nébo et Chiépo.

La majorité des eaux de surface de la zone d'étude sont claires à légèrement troubles, avec une turbidité moyenne de 27,17 NTU. Celles qui sont fortement troubles sont situées à proximité des sites miniers ou traversant des plantations. Kambiré et al., (2014), affirment que cette variation de la turbidité des eaux de surface pourrait être liée aux caractéristiques de la zone d'étude mais également à la pression anthropique autour des rivières.

Concernant les hydrofaciès, les eaux de la zone d'étude appartiennent au faciès Chloruré calcique et magnésien puis bicarbonaté calcique et magnésien. La prédominance du faciès chloruré calcique et magnésien dans les eaux du département est dû à la faible hétérogénéité lithologique du département. Ce résultat est en conformité avec celui obtenu par Kouassi et al., (2010) dans la région du N'Zi-Comoé, puis Yao., 2018 réalisés dans le domaine de la chimie des eaux à proximité de la mine d'or d'Afema.

Cette minéralisation a également été mis en évidence dans cette étude à partir de l'analyse statistique multivariée (ACP). L'Analyse en Composantes Principales Normée (ACPN) a donné des indications sur l'origine de la minéralisation des eaux du département. Celle-ci révèle que, la minéralisation des eaux est contrôlée par les activités anthropiques menées autour des points d'eau et la nature des formations géologiques présentes dans le département. En effet, la minéralisation des eaux est contrôlée par les produits d'altération (lessivage des altérites) qui peuvent se retrouver dans les cours d'eau par le phénomène de ruisselement (Megersa et al.,2015). 


\section{Conclusion}

La présente étude a permis de mettre en évidence les caractéristiques physico-chimiques des eaux de surface du département de Divo. Les résultats montrent que les eaux sont non agressives. Les eaux sont moyennement minéralisées avec une conductivité comprise entre 183,2 et $1909 \mu \mathrm{S} / \mathrm{cm}$, avec une moyenne de $608,4 \mu \mathrm{S} / \mathrm{cm}$. Les eaux à proximité des sites d'exploitation industriels sont les plus minéralisées. Les points d'eau à forte minéralisation sont HR1, HR2, HR3, HR4 et HR5 situés respectivement : à proximité d'un site minier, rivière à $200 \mathrm{~m}$ d'un pit utilisée par les orpailleurs en batée, rivière sortant de la mine à Hiré située à $100 \mathrm{~m}$ du pit et eaux à moins de $70 \mathrm{~m}$ du pit charpelle à Hiré, utilisée pour les Bovins. Les échantillons d'eau contiennent de fortes teneurs en nitrates, les teneurs varient de 1,2 à 164,8 mg/l avec une moyenne de 15,07 mg/l. Les eaux les plus influencées par les nitrates sont celles situées à proximité des sites d'exploitation minière d'Hiré et d'Agbaou (HR2 et HR5 situés respectivement : à proximité de la mine d'Akisso et rivière à $200 \mathrm{~m}$ d'un autre site). La prédominance du faciès chloruré calcique et magnésien dans les eaux du département est dû à la faible hétérogénéité lithologique du département. L'ACPN montre que la minéralisation des eaux est influencée par les activités anthropiques (le groupement est constitué par : la $\mathrm{CE}$, les cations $\left(\mathrm{Na}^{+}, \mathrm{Ca}^{2+}\right.$ et $\left.\mathrm{K}^{+}\right)$, les anions $\left(\mathrm{Cl}^{-}, \mathrm{HCO}_{3}^{-}, \mathrm{SO}_{4}{ }^{2-}\right)$ et $\mathrm{NO}_{3}{ }^{-}$dans sa partie négative. La présence du nitrate $\left(\mathrm{NO}_{3}{ }^{-}\right)$dans ce groupement a une origine superficielle et témoigne d'une pollution anthropique. Cela explique une minéralisation liée aux activités industrielles du département produit à la surface du sol, puis entraînées avec les eaux de ruissellement. Ces résultats mettent en relief la forte pression anthropique sur la qualité des eaux de surface du département de Divo. Des études plus détaillées sur les eaux et les types d'engrais, puis les produits utilisés en général lors des activités minières dans le département étayeront ces présomptions. En attendant, il est évident que ces eaux ne conviennent pas à la consommation humaine. Leur consommation sans traitement préalable expose la population à des risques pour la santé.

\section{Remerciements}

Les auteurs remercient le laboratoire mixte international LMI MINERWA, le réseau international AMEDEE, le Laboratoire des Sciences du Sol, de l'Eau et de Géomateriaux (LSSEG) de l'Université Félix Houphouët Boigny d'Abidjan et le laboratoire d'analyse de l'IRD de l'Université Nangui Abroguoa pour l'analyse des données.

\section{References:}

1. Adiaffi B, Marlin C, Oga YM-S, Massault M, Nauret A, Biemi J. 2009. Palaeo climatic and deforestation effect on the coastal fresh 
groundwater resources of SE Ivory Coast from isotopic and chemical evidence. J. Hydrol., 369: 130-141.

2. Adjagodo A., Agassounon D. T. M., Kelomè N.C., Vissin E.W., Agbossou E. (2017). Pollution Physique et Bactériologique de l'eau du fleuve dans la Basse Vallée de l'Ouémé pendant les périodes de Basses et Hautes eaux au Bénin. European Scientific Journal, Vol.13, No.33, pp. $1857-7881$.

3. Ahoussi K. E., Soro N., Koffi Y. B., Soro G., Biemi J. (2010). Origine de la minéralisation des eaux des aquifères discontinus sous couvert forestier de la zone Sud de la Côte d'Ivoire : cas de la région d'Abidjan-Agboville. International Journal of Biological and Chemical Sciences, Vol.4, N³, pp. 782-797.

4. Ahoussi K. E. (2008). Évaluation quantitative et qualitative des ressources en eau dans le Sud de la Côte d'Ivoire. Application de l'hydrochimie et des isotopes de l'environnement à l'étude des aquifères continus et discontinus de la région d'Abidjan-Agboville. Thèse de Doctorat de l'Université de Cocody-Abidjan (Côte d'Ivoire), $270 \mathrm{p}$.

5. Ahoussi Kouassi Ernest, Noël Keiba KEUMEAN, Michel Amani KOUASSI et Blaise Yao KOFFI. Etude des caractéristiques hydrogéochimiques et microbiologiques des eaux de consommation de la zone périurbaine de la ville de Man : cas du village de Kpangouin (Côte d'Ivoire), Int. J. Biol. Chem. Sci. 11(6) : 3018-3033, December 2018.

6. Akatumbila L., Mabiala M., Lubini A., Pwema K., Musibono E. A. 2016. Contribution à l'évaluation de la qualité physico-chimique de l'eau : cas de la rivière urbaine Gombe de Kinshasa/République Démocratique du Congo. Larhyss Journal, n²6, pp. 7-29.

7. Arrêté du 11 janvier 2007 relatif aux limites et références de qualité des eaux brutes et des eaux destinées à la consommation humaine mentionnées aux articles R. 1321-2, R. 1321-3, R. 1321-7 et R. 132138 du code de la santé publique.

8. Bamba Y. (2012). Evaluation des impacts de l'exploitation de la mine d'or de Bonikro sur les ressources en eau. Mémoire de master en ingénierie de l'eau et de l'environnement, 2IE(Ouagadougou), 55 p.

9. Bashir E., Huda S. N., Naseem S., Hamza S., Kaleem M. (2017). Geochemistry and quality parameters of dug and tube well water of Khipro, District Sanghar, Sindh, Pakistan. Applied Water Science 7 : pp 1645-1655. https://doi.org/10.1007/s13201-015-0316-9.

10. Biemi J. (1992). Contribution à l'étude géologique, hydrogéologique et par télédétection des bassins versants subsahéliens du socle précambrien d'Afrique de l'Ouest : Hydrostructurale, 
Hydrodynamique, Hydrochimie et Isotopie des aquifères discontinus de sillons et aires granitiques de la haute Marahoué (Côte d'Ivoire). Thèse d'État ès Sciences naturelles. Université de Cocody, Abidjan. 493p.

11. Chaouay A., Okhrib R., Hilali M., Bazzi L., Chahid A., Khiri F. (2016). Contribution à l'étude de l'analyse physico-chimique et de la contamination métallique de l'eau de mer du littoral d'Agadir (Sud du Maroc). Journal of Materials and Environmental Sciences, 7 (8), pp. 2748-2759.

12. Consortium MINEO. (2000) «Examen de l'impact environnemental et social potentiel de l'exploitation minière »http://www2. brgm.fr/mineo/User Need/IMPACTS. pdf.

13. Dudka S., Adriano Dc. (1997). Environmental of impact metal ore mining and prosessing : A Review, Journal of Environmental Quality, $26: 590-602$.

14. Durucan S., Korre A., Munoz-Melendez G. (2006). Mining life cycle modelling: a cradle-to gate approach to environmental management in the minerals industry. Journal of Cleaner Production (14), pp. 10571070.

15. Eblin, A. P. G. Sombo Soro, N.Aka, O. Mbiré, N. Oro, (2014).Hydrochimie des eaux de surface de la région d'Adiaké (sudest côtier de la Côte d'Ivoire). Journal of Applied Biosciences 75 :6259-6271 ISSN 1997-5902 J.

16. Kambire O., Adingra A.A., Eblin S.G., Aka N., Kakou A.C. et Koffinevry R. (2014). Caractéristique des eaux d'une lagune Estuarienne de la Côte d'Ivoire : la lagune Aby ; Larhyss Journal, ISSN 1112-3680, n 20, pp.95-110.

17. Lakhili F., Benabdelhadi M., Bouderka N., Lahrach H., Lahrach A. (2015). Etude de la qualité physicochimique et de la contamination métallique des eaux de surface du bassin versant de Beht (Maroc). European Scientific Journal, vol.11, No.11, pp. 1857- 7431.

18. LokoS., Ahoussi K. E., Koffi Y. B., Kakou N. F., Kouassi A. M., Biemi J. (2013). Microbiological and physico-chemical quality of groundwater from artisanal sites of mining exploitation in the SouthWest of Côte d'Ivoire : case of the area of Hiré. International Journal of Scientific \& Engineering Research, Vol.4, N ${ }^{\circ}$, pp. 567-574.

19. Megersa O., Dinkaa, W. L., Julius M. N. (2015). Hydrochemical characterization of various surface water and groundwater resources available in Matahara areas, Fantalle Woreda of Oromiyaregion. Journal of Hydrology, 3, pp 444-456.

20. Ngouala M.M., Mbilou U.G., Tchoumou M., Samba-Kimbata M.J. (2016). Caractérisation eaux de surface - eaux souterraines dans 
l'aquifère côtier du bassin versant de la Loémé en République du Congo. Larhyss Journal, $\mathrm{n}^{\circ} 28$, pp. 237-256.

21. Omanović D. PiżetaI. Vukosav P. Kovács E. Franċsković-Bilinski S. and Tamás J. "Assessing element distribution and speciation in a stream at abandoned $\mathrm{Pb}-\mathrm{Zn}$ mining site by combining classical, in-situ DGT and modelling approaches," Sci. Total Environ., vol. 511, pp. 423-434, April 2015.

22. Othmani, M. A. Souissi, F. Silva, E. F. and Coynel, A. "Geochemistry and potential environmental impact of the mine tailings at Rosh Pinah, southern Namibia," Journal of African Earth Sciences, vol. 111, pp. 231-243, July 2015.

23. Ouverture à Abidjan de la Revue annuelle 2016 de la performance du portefeuille de projets financés par la Banque mondiale en Côte d'ivoire Publié le jeudi 28 avril 2016.

24. RODIER J. (2009). L'analyse de l'eau. Ed. Dunod Paris. ISBN: 9782-10054179-9.

25. Salifu M., Yidana S. M., Anim-Gyampo M., Appenteng M., Saka D., Aidoo F., Gampson E., Sarfo M. (2017). Hydrogeochemical and isotopic studies of groundwater in the middle voltaian aquifers of the Gushegu district of the North ernregion. Appl Water Sci (2017) 7:1117-1129. DOI 10.1007/s13201-015-0348-1.

26. Tohouri P. (2012). Etude de la qualité physico-chimique des eaux de surface du bassin versant de la lagune Ono dans la Sous-préfecture de Bonoua (Sud-Est de la Côte d'Ivoire). Mémoire de Master de l'Université Félix Houphouët Boigny, 61 p.

27. Yao K. A. F. (2018) Développement d'une méthodologie pour une meilleure évaluation des impacts environnementaux de l'industrie extractive : cas de la mine d'or d'Afema (Sud-est de la Côte d'Ivoire). Thèse de doctorat, Université de Montpellier, France, 210 p.

28. Yapi Y.H.A., Dongui B.K., Trokourey A., Barima Y S.S., Essis Y., Atheba. P. (2014). Évaluation de la pollution métallique des eaux souterrain et de surface dans un environnement minier aurifère à Hiré (Cote d'Ivoire). Int. J. Biol. Chem. Sci.8 (3), pp1281-1289. 